\title{
LOS JUEGOS ANTROPOLOGICOS DE SAINT-LOUIS
}

\author{
DR. FABRICE DELSAHUT \\ Maître de conférence en Sciences et Techniques des Activités \\ Physiques et Sportives (STAPS) Université Paris 4 Sorbonne \\ (Paris - Île-de-France - France) \\ E-mail: f.delsahut@orange.fr
}

\begin{abstract}
RESUMEN
En 1904, en medio de la feria de la exposición universal de Saint-Louis, los Juegos olimpicos no pudieron escapar a la ascención de ideologías racistas del principios del siglo XX y contibuyeron a la discusión del mérito atlético de diferentes razas. Los organisadores prepararon competiciones especiales que llamaron en esta ocasión «Jornadas antropológicas» reservadas a los que la América segregacionista del momento consideraba como primitivos. El impacto de los estudios sobre las razas, sobre la manera de pensar en el deporte no fue, al contrario de lo que se esforzaron hacer creer los diferentes presidentes del C.O.I en el curso de las siguientes decadas, un simple avatar del movimiento olimpico. Veremos la influencía de este espectáculo atletico interracial en los Estados Unidos pero tambien en el mundo, particularmente a través del papel de la prensa. Las jornadas antropológicas permitieron indudablemente elaborar una cierta percepción de la otricidad y limitaran la integración de los pueblos autóctonas en la maquina deportiva mundial.
\end{abstract}

PALABRAS CLAVE: Antropología; jornadas antropológicas; juegos olimpicos; deporte. 
Despues de un siglo XIX rico en exibiciones antropo-zoologicas en Europa y en los Estados Unidos (sobre todo durante la Exposición universal de Chicago en I 893 o la de Bufalo en 1901), los Americanos organizarón en 1904, une nueva exposición universal en Saint Louis (Missouri). Celebrando la compra en 1803 de la Luisiana, la exposoción recorria unos 634 hectareas en el centro de Saint Louis e incluia mas de 200 edificios celebrando el poder de la ciencia y de la tecnologia a traves de las ultimas invenciones, de la electricidad al automobil, del avion al fonogramo, del cinema al radium. David R. Francis, presidente de la exposición, expuso claramente en su discurso de abertura que la Feria "para las generaciones futuras, sera un hito en el cumplimiento y el progreso humano". A lo mejor, la celebraciones reflejaban la mas finas qualidades humanas como el espiritú de empresa, la curiodidad, la inteligencia y el exito. A lo peor, las manifestaciones intentaban ordenar el mundo y sus poblaciones definiendo cuales eran superiores y cuales no eran civilzadas. Esta tercera feria internacional amerciana tenia como objectivo de contribuir a la educacion del "ciudadano modelo". Asi, la enseñanza tenia una grande importancia, para justificar la expansion colonial. Durante el siglo XIX, los Estados Unidos extendierón su soberania de los Caribe hasta el Pacifico, pensando que era su "destino manifesto" de controlar el continente de un oceano hasta el otro. La sección antropologica no estaba solamente ahi para ilustrar los sueños exoticos de un punto de vista europeo, como fue el caso en Paris en 1900, pero para demostrar lo que eran la desesperante primitividad y el barbarismo de esos pueblos que de ahora en adelante podrian tener ayuda con las escuelas del gobierno americano. Entre el 30 de abril y el I ro de diciembre, mas de 19 millones de visitantes vinieron a la Exposicion.

Los Juegos Olimpicos fueron englobados en el cuadro de las fiestas de la Exposición universal. Pierre de Coubertin tenia miedo en cuanto al buen desarollo, al impacto y a la originalidad de estas olimpiadas en el suelo americano. Los echos le dieron razon. El movimiento olimpico era todavia joven y sus miembros no prestaron sufisamente cuidado a las derivas que se desarollaron alli.

Las competiciones deportivas fueron dispersadas sobre mas de cuatro meses y medio, perdidas en el caos de la Exposicion. Ademas, estos Juegos Olimpicos fueron precedidos, quince dias antes, por "Jornadas antropologicas" durante las cuales los organizadores habian organizado competiciones especiales reservadas a los que los Americanos xenófobos en ese entonces consideravan como "primitivos".

En este contexto se enfrentarón Crow, Sioux, Pawnee, Navajo, Chippewa y "otros pueblos de los Estados Unidos", Ainu del Japon, Cocopa "de Baja California 
en Mexico", Sirios de Beyrouth, "Patagones de America del sur”, "Zulus y Pigmeos de Africa", y, de las Filipinas, Moros, Negritos, y lgorots, repartidos en ocho "grupos culturales" diferentes'. Estos "extraños" competidores - unos cientos van a participar a a los Olimpicos salvajes - porveniendo de los dos miles indigenas exhibidos en el marco de la Luisiana Purschase Exposition 2 ${ }^{2}$. Los antropologos, para la publicidad de estas jornadas, hasta habian convencidos el legendario jefe de los Indios Apaches, Geronimo, de aparecer durante la exibicion.

The anthropological ethletic meet hacia en realidad eco al programa de la Exposición, como teatro donde se inventaba la alteridad "exotica", un espacio de teorisación de las jerarquias raciales y el lugar donde estaban puesto de relieve estas desigualdades, designando asi los pueblos colonizados y colonizables.

Si el objetivo reconocido era verificar las reales capacidades fisicas de los "indígenas" y señalar al mundo la superioridad de la raza blanca, el estudio aprofondido de los medios de comunicación locales, nacionales e internacionales, particularmente los que fueron conservados por el Missouri Historical Society, muestran una voluntad mas implicita de señalar la superioridad de la raza blanca sobre los salvajes. Se repartieron entonces, a merced de los discursos antropologicos, las razas en la escala evolucionista. El deporte, como producto cultural de las naciones dominantes, se volvio aqui en un instrumento de socialización diferenciada de primera importancia y contribuyo sin saberlo a completar el proceso de la hominizacion.

Las busquedas llevadas plantean tambien la cuestion del papel de los medios de comunicación durante estas jornadas y sobre todo en la difusión de las teorias raciales de ese entonces. Simple testomonios o braso secular de una ciencia encantada por la voluntad de demostrar la superioridad del hombre blanco sobre todas las otras razas, esta difusión mediatica tuvo una influencia no despreciable sobre el porvenir deportivo de los pueblos otoctonos pero tambien sobre el de la joven nacion americana.

Estos "Juegos olimpicos especiales" apartenecen desde entonces a la historia del deporte y no representan, al contrario de lo que se esfuerzarón de hacer créer los diferentes presidentes del Comité Olimpico Inernacional durante las decadas siguientes, un avatar desgraciado del movimiento, indigno de la historia olimpinca.

I. Los Africanos, los Asiaticos, los Filipinos, los Aïnus, los Tehuelche o Indios "patagones", los Cocopas, Los Pueblos e Indios americanizados. Ver a propósito los diferentes "tipos culturales y físicos" describidos por David R. Francis (19/3).

2. En el contexto de las festividades, una exposición sobre "la guerra de los Boers" fue presentada. Dos autóctonos, miembros del pueblo Tswana, Lentauw (de su verdadero nombre Len Tau) y Yamasani (Jan Mashianià, habiendo juntado la South African Boer War Exhibition Company en Pretoria, fueron convidados a la exposición pero también a las jornadas antropológicas y al maratón olímpico. 
La organización de los Anthropological Days fue confiada a los departamentos de Antropologia y de Cultura fisica, asi que al Field Museum de Chicago. Entre antropologia y cultura fisica, estos dias de 1904 van a permitir de asegurar el lienzo de esa organizacin bicefala extraña. Esta doble organización esta a la origen de ciertas ambuguidades sobre la analysis de los resultados. Dos logicas vinieron cruzar el camino de las pruebas. Para John E. Sullivan, secretario de la poderosa e influente Union de los Atletas Aficionados, jefe del departamento de cultura fisica y director de los Juegos Olimpicos, estos juegos tenian como vocación de echar a perder los rumores, segun el, infundadas sobre las capacidades atleticas de los "salvajes" y preservar la integridad de los Juegos Olimpicos. Los previsibles fracasos deportivos de los indígenas anunciaban, a sus ojos, el futuro exitó de los atletas americanos en los Juegos ${ }^{3}$.

Para William J. McGee, primer Presidente de la American Anthropological Association y administrador del departamento antropoligicó de la exposición, si los Nativos disponian de verdaderos potenciales, entonces solo la antropologia, y sus diversos campos de estudio, serian capaz de analyzarlo y explicarlo.

La idea de los Specials Olympics fue suggerida a McGee por Sullivan. Aquel vio en los diferentes articulos de prensa venteando las predisposiciones fisicas de los Nativos, una ofensa indirecta a la existencia de su departamento y una posible vuelta a discutir de la superioridad atletica "natural" de los deportistas americanos "blancos".

Los pueblos nativos representaban también una buena publicidad indirecta para los Juegos olímpicos en tanto que las ventas de billetes estaban bajas y que los salvajes disfrutaban de una cierta popularidad en la Exposicion.

Esta suggestión vino a punto para McGee, Robert S. Woodworth (professor de sicologia en la Universidad de Columbia) y su estudiante Frank G. Bruner, que buscaban nuevos campos de experimentación antropometricas visto que tenian dificultades a obtener la permision de varios nativos para efectuar testos y producir los nuevos encuentros cientificos prometidos. La mayoria, por ejemplo, no quisieron subir en los arboles descalzos solo par medir el tiempo que tomaban. Ademas, solo un tierzo quizo ser fotografiado. Cuando Sullivan vino con la idea de "Juegos

3. Sobre ochenta pruebas consideradas generalmente como parte del programa olímpico, la mitad solamente contenía por lo menos un atleta no de origen de los Estados unidos. Sobre los seis ciento ochenta y un atleta presente, quinientos veinte y cinco eran Americanos. Los ciento cincuenta otros eran repartidos en once nacionalidades diferentes ósea el cuota más bajo de toda la historia de los Juegos. Los Americanos ganaron setenta y nueve medallas de oro sobre las noventa y cuatro en competición. 
Olimpicos Especiales" bajo los auspicios comunes de los dos departamentos, McGee y WoodWorth decidieron concentrar principalmente los testos antropometricos sobre la fuerza, la velocidad y la resistencia durante eventos deportivos. Sullivan llamo el evento "Jornadas antropologicas" en honor al doctro McGee. Segun el, estas jornadas iban a permetir enfrentar la realidad y las suposiciones, y sobre todo asegurar indirectamente la promoción de los verdaderos Juego olimpicos.

\section{LA HABILIDAD DEPORTIVA EN EL PROGRAMA}

Dos dias estaban previstas en el programa, a saber el 12 y 13 de agsoto de 1904. Durante la primera, los participantes concurieron entre ellos dentro de grupos tribales predefinidos, en diciplinas resultadas del programa olimpico. Desde las primeras pruebas, se vio que los resultados iban a ser muy abajo de las esperanzas. La prueba de 100 yards fue ganada por George Ments, presentado como un "Americanized Indian" con un tiempo ( I l"4) relativamente mediocre. El ultimo era un Pigmeo Mbuti, llamado Shamba, en 14"3 I. El autor del informe noto, no sin perfidia, que el joven Africano cierto llevaba una vida al gran aire, cazaba, corria, nadaba, saltaba y utilizaba el arco y la lanza, y que sa vida podia ser qualificada de "naturalmente atletica", pero que, a pesar de todo, necesitaba mas de catorze sugundos para parcourir cien yards y que cualquier atleta americano lo hubiera vencido con por lo menos catorze yars de diferencia.

El segundo dia revelo los "salvajes" bajo un mejor dia visto que le dieron la oportunidad de mostrar lo que eran capaz de cumplir en ciertas de sus "practicas ancestrales". La merjor realización fue la de un lgorot, originario de las Filipinas, que subio arriba de una percha de quince metros en veinte segundos. La jabalina y el tiro al arco, al contrario, deceptionarón los observadores que esperaban encontrar en estas pruebas, la hipotetica habilidad de los "pueblos salvajes". Los Coropas, originarios de Mexico, dieron al fin del dia una demostracion de su juego Shinny, el ancetro del hockey sobre grama. Pero fue juzgado "sin interez" en cuanto al trabajo de equipo inexistente y de "intentos puramente individuales de parte de los jugadores". Segun Sullivan, enseñó cuanto las competencias de organización eran arcáicas y la coperación imposible. En conclusión, revelaba la ausencia de inteligencia necesaria a la construcción de une equipo y a la cordinación de los esfuerzos de cada uno para alcanzar la victoria.

Por fin los Pigmeos cedieron a su "juego favorido", lanzar barro, que fue comparado a las batallas de bolas de nieves de los niños americanos. La demostración fue muy apreciada por el publico y se noto sus aptitudes a esquivar, lanzar y correr. 
Los Juegos Tribales hicieron ver al mundo la superioridad insolente de la "raza de tipo caucazico" sobre las minoridades etnicas. El informe Spalding lo muestra, con una cierta ambiguidad, y ofrece una verdadera lección de "etnografia popular" (MCALOON, 1981, p. 134). De manera general, se burlaron, de las realizaciones en las diferentes pruebas ${ }^{4}$ enfrente de las potencialidades esperadas o mejor dicho "fantasmadas" de parte de los organizadores. Las realizaciones de los Patagones, cuya estadura dejaba esperar para los observadores realizaciones notables en lanzamiento de peso, fueron cualificadas de "realización rediculamente debil que asombro todos los testimonios" (SULLIVAN, 1905, p. 25I). El informe insistio tambien sobre el hecho que el campión americano Ray Ewry saltaba mas lejo sin arranque que cualquier "salvaje" con arranque. Las proezas relativas de los Aïnus hizo que tubierion que notifiar en el informe que aquellos tenian todavia enormes "esfuerzos" a cumplir en el camino del desarollo corporal. Por otra parte, se puede leer que "se puede probablemente decir, sin temer contradicción, que nunca antes en la historia del deporte en el mundo, tales realizaciones tan pobres habian sidas registradas en el lanzamiento de peso" (SULLIVAN, 1905, p. 253). John E. Sullivan no encontro las palabras bastante duras para expresar su decepción no tanto en los resultados que esperaban, sino en las atitudes de los indígenas durante las pruebas. Lo que Nancy Parezo resumo por este comentario :

Los participantes nativos, por supuesto, no realizaban que habian decepcionado cualquier. Ademas, como puede ser que no sabian que sus realizaciones eran utilizadas para sacar conclusiones sobre las capacidades atleticas de todos los pueblos "primitivos" o que su supuesta posición en una hierarquia evolucionista seria afirmada de nuevo con la competición falseada, la logica defectuosa, o de la generalidades de Sullivan (PAREZO, 2008, p. I02).

McGee nego esta condanación. Explico que estas "contra realizaciones" eran debida a una "falta de educación", o simplemente de previa practica de los diferentes deportes por los atletas indígenas. Estas explicaciones no satisfacieron del todo los observadores y el publico. Ademas, subrayo la extrema "amabilidad" de los Aïnus, los "salvajes" los mas educados que el autor he encontrado, y su compromiso activo en todas las actividades deportistas. Al contrario, los Pigmeos, cuyo compromiso ha sido obtenido en contra algunas sandias, no recibieron el mismo testimonio y fueron presentados como "maliciosos" et no tomaban nada en serio, menos sus juegos tradicionales.

4. 100 yards, putting 16-lb ?shot, 440 yards run, Runnig broad jump, Throwing bolos, Throwing baseball, Throwing 56-lb weight, 120 yards hurdle race, Throwing the ball for accuracy, I mile run, Throwing Javelin (25-foot distance), 120 yard hurdle race, Climbing pole, Running high jump, Archery, 440 yard run, Tug-of-war. 
El Doctor McGee noto tambien el hecho que los indígenas no se comprometierón en las pruebas impuestas por el simple hecho que "algunos no sabian que uno espera lo mejor posible de ellos" (SULLIVAN, I905, p. 257). El informe subrayo tambien el hecho que un dia suplementario hubiera sido necesario para permitir a los traductores de explicar las pruebas a los participantes. McGee quizo, sin gran éxito popular, organizar de nuevo las pruebas durante el mes de septiembre ${ }^{5}$.

Las pruebas eliminatorias de los 100 yards fueron muy reveladoras a este respeto. El principio de la carrera era siñalada por un disparo y la linea de llegada era materialisada por una cinta tendida de travez a nivel de pecho que el vencedor rompia. Eso necesito mucho coraje y paciencia a los oficiales para explicar a la decena de corredores que tenian que empezar al disparo y parrarse antes de la cinta de llegada. El informe hasta noto que el Dr. Martin Delaney, de la Universidad de Saint-Louis, tuvo que enseñar a los competidores los que uno esperaba de ellos.

Falta de compromiso, incompresion de las pruebas, entrenamiento inadecuado o inexistente y participación forzada son tantas razones de limitación de lo resultados de los atletas presentes. Consta que estas razones, bien identificadas por todos, fueron minoradas despues para validar la tesis de la superioridad racial de los blancos. Los resultados, falsificados, probaba "de manera concluyente que el salvaje habia sido un hombre previamente sobrestimado de punto de vista deportista" (SULLIVAN, 1905, p. 257). Y Sullivan añadio: "de punto de vista cientifico, eso probia de manera concluyente que el salvaje o extranjero no era el igual del hombre blanco" (WORLD'S, 1904, p. 50). La lección fue brillante:

Los cientificos van a referirse a esto durante muchos años despues. Fue una grande leccion. Los lectores y autores tendran que olvidar todas la referencias a las capacidades atleticas naturales de los salvajes, a menos que puedan dar la pruebas de sus presumidas hazañas (SULLIVAN, 1905, p. 259).

5. Para McGee, las verdaderas decepciones de las jornadas provenían de hecho que no habían podido educar la gente, y Sullivan, sobre el valor cientíico de la Antropología y que no habían ganado dinero para su departamento. Decidió entonces organizar una segunda competición en septiembre para "dar al público una oportunidad de ver lo mejor de las tribus primitivas concurrir en juegos modernos y nativos de fuerza, resistencia y agilidad" (PAREZO, 2008, p. 103). Esperaba obtener una ayuda financiera sin éxito, entonces tuvo que tomar en sus propias reservas. Los grupos empezaron a entrenarse bajo la dirección de Simms e hicieron ensayos. Los grupos se enfrentaron, concurriendo los unos contra los otros, raza por raza, utilizando el mismo modelo que antes. McGee llamo el evento "Encuentros antropológicos" porque Sullivan no quiso utilizar el término "Special Olympics". El evento de septiembre fue un éxito relativo pero a pesar de todo, pudimos notar que un periodista del St. Louis Globe-Democrat escribió que "el encuentro fue un éxito a todo punto de vista, y sirvió de ejemplo sobre los que los hombres de color eran capaz de hacer con entrenamiento" (MOROS, 1904) 
Si las conclusiones de Sullivan y McGee son identicas, los medios para alcanzar los resultados son bien diferentes. El contexto, sus responsabilidades y sus fuertes compromisos en sus departamentos respetivos no podian llegar a concluciones diferamente sobre el resultados des estas jornadas. Sullivan fue incapaz de razonar de otra manera que con el paradigmo deportista y comparo todos los resultados fisicos fuera del contexto de los autoctonos a los resultados deportistas caros a los Juegos olimpicos. Critico en primer lugar las teorias de McGee alegando que esas no tenian cualquier carácter cientifico y solo servian para informar el espectador. En cuanto a los salvajes, concluyo que los resultados no podian ser mejor por falta genetico de capacidades intelectuales ligada a la comprension y de capacidades motrices ligada a la adaptación. McGee templo un poco estas decalraciones y siguio un tiempo las de Stephen Simms, el conservador del Field Museum que tuvo que notar todos los dias las pruebas y que critico sus condiciones y su carácter aculturador.

\section{LAS PROEZAS DE LOS SALVAJES.}

Desde 1903, McGee fue solicitado para concebir y administrar el departamento antropológico de la exposición. Gracias a los "life and movements exhibits" clase de reconstitución de los modos de vida y de los medios ambientes, esperaba que el contacto directo entre los Americanos y los "pueblos indígenas" seria "el mejor disolvente de la hostilidad y desconfianza entre las personas y los pueblos". Quería también mostrar la importancia de un saber Antropológico y puso todo en ejecución para que este último sea desarrollado durante esos días. Para Nancy Parezo, esta postura en frente de los pueblos indígenas es totalmente diferente:

su objetivo era de visualizar la evolución unilineal, combatir los estereotipos al motivo de los pueblos autóctonos y reunir los indígenas en un solo y mismo lugar de manera que los científicos americanos y europeos puedan prolongar sus conocimientos raciales con observaciones sistemáticas de primera mano, estudios antropométricos y etnográficos (Unpublished document) ${ }^{6}$

6. PAREZO, N. Anthropology Days, Fabricating and Testing Racial Strength and Endurance at the 1904 Louisiana Purchase Exposition. In: THIRD ANNUAL MEETING OF THE CULTURAL STUDIES ASSOCIATION, Tucson: University of Arizona, April 2005. Unpublished document 
Creemos desde años, a partir de las declaraciones hechas por los que estaban sensatos saber ${ }^{7}$, y a partir de artículos de periódicos y libros que el salvaje medio era ligero a la carrera, de robusta constitución, preciso con un arco y una flecha y experto en el lanzamiento de piedra y que algunos, particularmente los Patagones, eran reconocidos par su alta estatura, y fuerza, y por caso de la vida particular que muchos de ellos llevaban, fueron llamados atletas naturales [.... Conocemos las maravillosas calidades de los Indios como corredores, la resistencia de los Kaffir, y las capacidades generales de los salvajes durante hazañas atléticas, pero los eventos de Saint-Louis refutaron esas historias (SULLIVAN, 1905, p. 249).

La idea claramente entrevista en este relato era de verificar con un punto de vista científico las calidades físicas dichas naturales de los Autóctonos y de confrontarlos al mito de la superioridad sensori-motriz de las "gentes de color" haciendo disputarse pruebas atléticas anglo-americanas así como algunos juegos tradicionales autóctonos. Los resultados eran entonces comparados a las capacidades registradas por los Blancos. Por ejemplo, una de las prestaciones más "perjudiciales" a los participantes autóctonos fue el partido de Shinny. Influenciado por las teorías psicológicas evolucionarias de G. Stanley Hall, Sullivan consideraba que los deportes de equipo eran ideales para forjar el moral y el físico. La capacidad de ser un jugador colectivo parecía como un requisito indispensable para una buena adaptación ciudadana a la sociedad moderna. Por extensión la capacidad de participar en deportes colectivos institucionales era un marcador fuerte de la facultad para un grupo étnico (indígenas pero también inmigrantes de Europa) a asimilar la civilización.

Además de las ciencias, símbolos y retoricas vinieron de manera más trivial completar la empresa de justificación de una jerarquización racial. Los Juegos Olímpicos de Saint Louis fueron los primeros durante los cuales medallas de oro, de plata y de bronce fueron atribuidas. Pero los organizadores no ofrecieron un tal honor a los "salvajes". Al lugar de una medalla, los ganadores recibieron una bandera americana que, como el himno nacional, instrumentalizaba el patriotismo americano y encontraba en el deporte la indispensable caja de resonancia susceptible de exaltar su nivel simbólico.

7. Por ejemplo, el Doctor Blanchi expone desde 1902 en un relato siguiendo la Exposición universal internacional de 1900 en Paris las capacidades atléticas de los Negros. "Entre los negros donde la educación física de los órganos se hace inconscientemente desde el nacimiento, los órganos internos tienen las mejores condiciones y se acercan de los de nuestros mejores corredores. Por otra parte, es por esta condición fisiológica del negro, que se debe su resistencia porfiado al corredor que le da la capacidad de seguir un jinete durante un día entero sin cansarse de una manera extraordinaria" (BOULARAND, 1999, p. 147) 


\section{LOS MEDIAS O EL PODER DE LAS REPRESENTACIONES DEL OTRO}

Los simples hechos relativos a los juegos antropológicos, a saber el desarrollo de las pruebas, y más generalmente su ambiente acogedor, no bastan a subrayar la construcción de un imaginario social sobre el otro. Por eso hay que estudiar los escritos de esta época y especialmente los periódicos relatando y comentando los hechos. En paralela de las exhibiciones y competiciones, un racismo popular se desplego en la prensa mayor y en la opinión pública como tela de fundo de una justificación científica de la desigualdad de las razas humanas y de la conquista colonial.

Los medias nacionales relevaron los propósitos de las ciencias del deporte y de la antropología naciente, poniéndose así al servicio de una jerarquización racial y de un derecho a la colonización especialmente deportista. Todos los medias, de los periódicos locales - como el St.Louis Republic o el St. Louis Post-Dispatch - a las publicaciones a carácter "científico"- como la North American Review o Ciencia - se esforzaron presentar las populaciones exóticas - y muy particularmente las sometidas a la conquista - como vestigios de los primeros estados de la humanidad. Ellos contribuyeron también al nacimiento de una nación americana poniendo el deporte al centro de su constitución. Su papel fue importante en la conciencia para los americanos de la superioridad de sus cuerpos, de sus modos de vida y de su cultura.

\section{UNO DOBLE PUESTA COYUNTURAL}

El análisis de los artículos de prensa relativos a los "Juegos Especiales" enseña la doble puesta coyuntural que han llevado. Han tenido por efecto de posicionar el tipo caucásico y su modelo cultural al nivel planetario y de proponer un nuevo ideal de sociedad, a través el deporte y la cultura física, fiador del renuevo nacional, para la mayoría. Como lo declara el presidente Theodore Jr al St.Louis Post-Dispatch: "he aprendido y visto más durante que yo estaba en la Feria que durante todo un año a viajar. Cada muchacho tendría que preguntar a su padre de dejarlo venir aquí y quedarse por los menos una semana" (A BOY'S, 6 de agosto 1904).

La prensa fue el terreno de la expresión y difusión de la ideología racialista de la política americana, a través varios aspectos. En primer lugar ella aseguraba la creencia en una especie humana dividida en "razas humanas", que se tenía que ordenar. El vocabulario de estigmatización del salvajismo fue esforzado por una producción iconográfica frecuentemente violenta, acreditando la idea de una subhumanidad estancada, humanidad de los confines coloniales, a la frontera de la humanidad y de la animalidad. Las expresiones como "barbaros", "comedor de perros", "pueblos primitivos" o "extrañas razas de hombre", los calificativos 
como "Aïnu peludos" o "caníbales" (un subgrupo del grupo africano ${ }^{8}$ ) servían, según Matti Goksyr, a mantener una distancia social, una diferencia de estatuto entre los visitantes y los pueblos. "La gente juntada para el departamento de Antropología en Saint-Louis no fue expuesta como técnico o artesano, pero simplemente como monstro" (GOKSYR, 1990, p. 300). Esos calificativos no son finalmente que la imagen invertida de la ferocidad - bien real esta - de la conquista colonial ella-misma. En esta animalización, la transgresión de los valores y de las normas que constituyen, para América, la civilización es un elemento motor y permite legitimar la brutalidad de los conquistadores en animalizando los conquistados o en paso de serlo.

Ota Benga, un joven Pigmeo fue así expuesto al título de "eslabón faltando", porque llevando en él, marcas visibles de su animalidad. Al centro de esta crónica del extraño, de este museo vivo, los organizadores, en su preocupación de minar el mito del atleta natural, tenían que enfrentar al problema de la percepción romántica del "noble salvaje". Este último, viviendo bajo las leyes del instinto y de la sabiduría para adaptarse a los diferentes medios ambientes naturales, se opone al hombre moderno corrupto y alienado por una industrialización que estorba la expansión de sus deseos. Es este ideal tipo que debía, indirectamente, enfrentar la "raza blanca" durante esos dos días.

La prensa reacciono también abundantemente vulgarizando las declaraciones de los herederos de una antropología victoriana para asentar el gran poder de la ciencia y especialmente la "ciencia del hombre" única ciencia natural de las "razas humanas".

Contribuya por fin a la educación ideológica deportista del ciudadano como hilo principal que une todos los americanos. Los contra resultados de los autóctonos y los éxitos de los atletas americanos en los Juegos olímpicos permitían a la prensa de enseñar la polivalencia de la ideología vehiculada por los deportes. La inspiración puritana (purificarse por el esfuerzo y el sufrimiento) todo como la inspiración darwinista (siempre pelearse para ser el más fuerte) y la inspiración progresista (alcanzar la salud, alegría y paz) construyen desde 1904 los valores comunes.

\section{UNA INFLUENCIA RELATIVA?}

Si hay que relativizar la influencia de este espectáculo atlético interracial fuera de los Estados-Unidos, este último ha seguramente permitido de reforzar una cierta percepción de la alteridad. Aportó una legitimidad científica al racismo y algunos hicieron referencia mucho tiempo. Una enciclopedia deportista sueca editada en 1943 recupero los resultados de las jornadas para concluir que "no es posible hacer de Negros africanos estrellas deportivas". Una tal posición fue adoptada antes

8. Uno de los Africanos fue llamado « Caníbal » por los organizadores en el registro del base-ball. 
por el investigador alemán Arthur Grix relativo a la falta de inteligencia táctica de los corredores Tarahumaras de México (GOKSYR, 1990, p. 303). Confirmando la alteridad, las jornadas antropológicas marcaron los espíritus populares y aferraron sustancialmente en la idiosincrasia americana la legitimidad del hecho colonial y el orgullo del gran pueblo colonizador. La mayoría de los arquetipos encenizados durante la Exposición universal y los Juegos antropológicos dibujaron la raíz de un inconsciente colectivo que durante el siglo tomo múltiples caras y que, hoy, es todavía indispensable de desconstruir. Las resurgencias fueron mucho más importantes y pérfidas que se puede pensar.

Hasta liberado del estrecho picota cientíico, el Otro tenía dificultad para alcanzar por fin su verdadera dimensión. "El espectro del pato", como lo defino Michel Dalloni (L'EQUIPE, 2000) no hace realmente soñar el espectador. La imagen de Eric Moussambani, el nadador de la Guinea Ecuatorial, forcejando contra las olas en el Aquatic Center de Homebushbay en Sídney durante los Juegos olímpicos de verano en 2000 dividió profundamente los enamorados del deporte así que la prensa. Para ciertos fue la representación de lo que los juegos pueden ofrecer de más bello: el desinterés. Para los otros no era más que una diversión. La prensa noto cínicamente que el campeón neerlandés Peter Van der Hoogenband había recorrido, en uno tiempo inferior de algunas segundas, el doble de distancia. En otros tiempos, en Saint-Louis, el Saint-Louis Globe Democrat comparaba el resultado de los Moros en natación a la de los "Patos en el agua" Más recientemente, en el 2007, el premio Nobel de Medicina, James Watson, declaro en el Sunday Times que la inteligencia de los Blancos, "no es la misma" que la de los africanos. Este regreso posible del "racismo científico" hace eco a la investigación biomédica pública que clasifica desde 200 I en los Estados-Unidos, los participantes para pruebas en grupos etno-raciales definidos por la administración?.

\section{CONCLUSIÓN}

Referente a las jornadas antropológicas, Pierre de Coubertin declaro: "En cuanto a esta mascarada ultrajante, se despojara todo naturalmente de sus oropeles cuando estos Negros, estos Rojos, estos Amarillos aprendieran a correr, a saltar, a lanzar y dejaran los Blancos atrás de ellos" (COUBERTIN, 1979, p. 43.). Estas palabras reconfortantes no fueron forzosamente proféticas. Estas jornadas han frenado, de un cierto modo, el acceso de las minoridades a los Juegos Olímpicos. Los buenos resultados de las "mitades civilizadas" a los Juegos Olímpicos de Saint-

9. Cf. "La tentación de la raza", Le Monde, Miércoles 3 I de octubre de 2007 
Louis fueron largamente pasados bajo el silencio al contrario de los de las jornadas. El equipo de basket-ball femenino del internado de Ft Shaw, las Ft. Shaw "Blues" derroto todos los equipos escolares euroamericanas y los clubs no profesionales que encontraron durante la Exposición. Estos hechos no fueron comentando en el Spalding's Athletic Almanac. "La capacidad deportiva no tenía ninguna relación con la raza, pero los poderosos guardias de los records no querían admitirlo- no mismo en el futuro." (PAREZO, 2008, p. I I3). Además, un corredor del Wisconsin llamado George Coleman Poage, revestido de los colores de su club de atletismo, el Milwaukee Athletic Club, gano dos medallas de bronce al 200 metros vallas y al 400 metros vallas. Fue así el primer atleta negro a ganar una medalla olímpica. Su resultado, aunque remarcado, no hizo la primera página de la prensa.

Al seguir las investigaciones llevadas en Saint-Louis y a los resultados de las jornadas, la aptitud deportiva de los "salvajes" siguió siendo una cuestión enteramente periférica durante muchos años. Coubertin, reticente al principio a la idea de la extensión de las prácticas atléticas a las clases pobres de las sociedades occidentales y, sobre todo a los "salvajes" de los países colonizados, cambio después radicalmente de opinión y afirmo que los Juegos olímpicos no eran la propiedad de ningún país ni ninguna raza (DELSAHUT, I999, p. 18-19). Esta perspectiva se concretizó progresivamente pero en las condiciones de una mundialización deportiva ávida de ganancias y uniformización. El olimpismo de Coubertin sobrevive desde ahora fuera de los Juegos olímpicos. Echados fuera del templo, mal representados o prohibidos de acceso para razones de imperativos politico-economicos, las Primeras Naciones han redefinido el esencial: deporte sin conciencia no es otra cosa que ruina del hombre. Esta definición esta al centro de los encuentros deportivos autóctonos como los World Eskimo-Indian Olympics o los Jogos dos Povos Indigenas de Amazonia en los cuales los juegos tradicionales permiten de encontrar de nuevo o perpetuar una cultura. El espíritu olímpico sopla desde ahora sobre las tierras del gran norte y de Amazonia.

\section{As jornadas antropológicas em Sant Louis}

RESUMO: Em 1904, como parte da Exposição Universal de SaintLouis, os Jogos Olímpicos não puderam escapar da ascensão de ideologias racistas de princípios do século vinte, de forma que estiveram no centro da discussão da potencialidade esportiva de diferentes raças. Forma preparadas competições especiais chamadas "Jornadas Antropológicas", destinadas àqueles que para a América segregacionista da época. O impacto dos estudos sobre raça sobre a maneira de pensar no esporte não foi, ao contrário do que os diferentes presidentes do COl se esforçaram em fazer crer no curso das décadas seguintes, um simples avatar do movimento olímpico. Veremos a influência deste espetáculo atlético interracial não apenas 
nos Estados Unidos, mas no mundo, particularmente no que se refere ao papel da imprensa. As Jornadas Antropológicas permitiram, indubitavelmente, elaborar uma certa perspectiva sobre a outricidade e limitaram a integração dos povos autóctones na máquina esportiva mundial.

PALAVRAS-CHAVE: Antropologia; Jornadas Antropológicas; Jogos Olímpicos; esporte.

\section{The anthropology days of Saint-Louis}

ABSTRACT: In 1904, in the Louisiana Purchase Exposition, the Olympic Games couldn't escape the rise in the racist ideologies of the 20th century and they contributed to the discussing of the athletic merit of the different races. Their organizers set up special competitions, called for the occasion "Anthropology Days ", reserved for those who were considered as primitives by the segregationist America of that time. The impact of the race studies on the ways of thinking sport, contrary to what the different chairmen of the IOC tried hard to make believe during the new decades, was not only an unfortunate moment in the history of the Olympic movement. We shall make some forecasts regarding the influence of that interracial athletic show in the United States but also in the world, in particular through the role of the press. The anthropology days surely allowed a certain perception of otherness and limited the integration of native peoples in the world sports fabric.

KEYWORDS: Anthropology; Anthropological Days; Olympic Games; sport.

\section{REFERENCIAS}

BOULARAND, H. Passé colonial et présent de l'immigration: Contribution à un nouveau regard sur l'éducation physique. In : GLEYSE J. L'Education physique au XXe siècle: approches historique et culturelle. Paris: Vigot, p. 141-158, 1999.

A BOY'S view of the fair. St. Louis Post-Dispatch, Saint Louis, 6 aug. 1904.

COUBERTIN, P. Olympic memoirs. Lausanne: International Olympic Committee, 1979.

DALLONI, M. Le spectre du toquard. L'Equipe, 20 septembre 2000.

DELSAHUT, F. Les hommes libres et l'olympe. Paris: L'Harmattan, 1999.

GOKSYR, M. "One certainly expected a great deal more from the savages": the anthropology days in St. Louis, 1904, and aftermath. The International Journal of The History of Sport, United Kingdon, v. 7, n. 2, p. 297-306, sept. 1990.

MACALOON, J. This great symbol: Pierre de Coubertin and the origins of the modern olympic games. Chicago: University of Chicago Press, 1981.

PAREZO, N.; FOWLER D. Anthropology goes to the fair: the 1904 Louisiana purchase exposition. Nebraska: University of Nebraska, 2007. 
MOROS win championship of philippine natives on track. St. Louis Globe-Democrat, Saint Louis, 16 sept. 1904.

PAREZO, N. A special olympics: testing racial strength and endurance at the 1904 Louisiana purchase exposition. In: BROWNELL S. The 1904 anthropology days and olympic games: sport, race, and american imperialism. Nebraska: University of Nebraska, 2008. p. 59- 126.

SULLIVAN, J. E. Anthropology days at the stadium, spalding's official athletic almanac, Special Olympic Number, v. 18, n. 217, p. 249-264, Jan. 1905.

WORLD'S FAIR BULLETIN, Saint Louis, v. 5, n. I I, sept. 1904.

Recebido: 31 ago. 2010 Aprovado: 01 fev. 201 I

Endereço para correspondência Fabrice Delsahut Université Paris IV Sorbonne - IUFM

10 rue Molitor 75016 Paris France 